\title{
Aspects of birth history and outcome in diplegics attending specialised educational facilities
}

Faith Bischof, Alan Rothberg, Ingrid Ratcliffe

Aim. We aimed to study functional mobility and visual performance in spastic diplegic children and adolescents attending specialised schools.

Methods. Spastic diplegia (SD) was confirmed by clinical examination. Birth and related history were added to explore relationships between $\mathrm{SD}$, birth weight (BW) and duration of pregnancy. Place of birth, BW, gestational age (GA) and length of hospital stay were obtained by means of parental recall. Outcome measures included the functional mobility scale (FMS) and Beery tests of visuomotor integration (VMI) and visual perception (VIS).

Results. Forty participants were included (age 7 years 5 months - 19 years 6 months). Term and preterm births were almost equally represented. Functional mobility assessments showed that 20 were walking independently in school and community settings and the remainder used walking aids or wheelchairs. There were no significant correlations between BW or GA and outcomes (FMS, VIS-z-scores or VMI-z-scores) and z-scores were low. VIS scores correlated significantly with chronological age $(p=0.024)$. There were also significant correlations between VIS and VMI scores and school grade appropriateness ( $p=0.004 ; p=0.027$, respectively).

Interpretation. Both term and preterm births were represented, and outcomes were similar regardless of GA. VIS and VMI were affected in both groups. Half of the group used assistive mobility devices and three-quarters were delayed in terms of their educational level. These problems require specialised teaching strategies, appropriate resources and a school environment that caters for mobility limitations.

S Afr Med J 2012;102(5):299-302.
We present one of several follow-up and outcome studies on children and adolescents with cerebral palsy (CP) by a small multidisciplinary group including practitioners in paediatrics, physiotherapy, occupational therapy, orthopaedic surgery and special education. Studies have reported on long-term outcome of various orthopaedic procedures and long bone fractures in residents of longterm care facilities. ${ }^{1-3}$ Recently the group focused on several outcomes in children and adolescents with a diagnosis of spastic diplegia (SD). The paediatricians asked whether in an era of rapid technological and

School of Therapeutic Sciences, Faculty of Health Sciences, University of the Witwatersrand, Johannesburg

Faith Bischof, MSc Physiotherapy, PhD

Alan Rothberg, MB BCh, PhD, FCPaed

Ingrid Ratcliffe, MSc Occupational Therapy, $\mathrm{PhD}$ therapeutic advances in neonatology, severe and complicated SD was being seen with the same frequency as in the past.

$\mathrm{CP}$ is a term covering a group of non-progressive motor impairment syndromes secondary to lesions of the brain that arise in the early stages of development. ${ }^{4}$ Diplegia is a subtype of bilateral spastic CP, also referred to as leg-dominated $\mathrm{CP}^{5}$ The lower limbs are more affected than the upper limbs and fine-motor function is usually impaired. The gait and mobility limitations in SD may be accompanied by cognitive deficits and corticovisual impairment. ${ }^{6}$ Diplegia is typically attributed to periventricular leucomalacia (PVL), which is necrosis of white matter in the internal capsule of the brain. The leucomalacia can be caused by intracranial haemorrhage or ischaemic infarction. ${ }^{6}$ Whereas PVL is usually considered to be primarily associated with prematurity (particularly extreme prematurity), it has also been demonstrated radiologically in term infants, producing motor deficits similar to those found in preterm infants. ${ }^{7-9}$

It seems reasonable to expect that significant developments in neonatology, such as the routine availability of artificial surfactant, would have led to improved short- and long-term outcomes in infants born after $1980 .^{10}$ However, the developments have also facilitated 
the survival of ever-smaller infants with a concomitantly higher propensity for neurological damage. ${ }^{11,12}$ This aspect is perhaps less of an issue in South Africa where, in the 1980s the public sector, which caters for the majority of South Africans, adopted a policy of non-ventilation of infants weighing $<1000 \mathrm{~g}$ at birth. This policy was necessary because of the limited number of neonatal intensive care beds and the extended ventilatory requirements of extremely preterm neonates (up to 3 months) that prevented admission of larger numbers of more mature neonates with better prognoses. ${ }^{13}$ Given this South African situation where neonatal care advances are predominantly directed towards neonates with better chances of intact survival, it would seem reasonable to anticipate a reduction in SD over time.

This question of frequency of SD since the 1980s was added to a developmental follow-up that had been planned to assess functional mobility of children and adolescents attending special education facilities, their educational level and the severity of visual perception (VIS) and visuomotor impairments (VMI). The study group included SD children with special educational needs resident in urban areas and with access to specialised schools. These schools offer adapted educational curricula, and therapy is provided on site.

\section{Methods}

The convenience sample included all the children and adolescents identified with SD who were attending four specialised schools in Johannesburg. Three of them were schools for learners with special educational needs (LSEN), catering for both learning and physical disabilities, while one school admitted children with moderate to severe intellectual disability. The three LSEN schools offered normal curricula up to grade 7, grade 9 and grade 12 respectively, as well as adapted curriculae. The fourth school offered limited skills training only. All the schools served families from a diverse range of socioeconomic backgrounds ranging from poor to affluent.

The diagnosis of diplegia was confirmed by complete and comprehensive clinical examination, focusing on the nature and distribution of the spasticity. Fifty subjects born between 1988 and 2000 were eligible for the study. Ethical approval was obtained from the Human Research Ethics Committee (Medical) of the University of the Witwatersrand.

Hospital records were not reviewed in the study. Birth history was obtained from parents, covering aspects that are unlikely to be forgotten over time. A questionnaire sent to the parents sought information including the duration of pregnancy, birth weight (BW), place of birth (public or private hospital) and length of the child's stay in hospital. In our experience, particularly in cases associated with postnatal problems and morbidity, these facts are fairly ingrained in parents' minds.

Activity limitation was determined by use of the functional mobility scale (FMS) which rates the amount of support required for walking distances of 5, 50 and 500 metres, representing mobility at home, at school and in the community. ${ }^{14}$ The Beery developmental test was used to measure VMI, and VIS was also measured. Results for VMI and VIS were expressed as z-scores, i.e. age-adjusted deviations from the mean. ${ }^{15}$ The VMI is based on a sample of 11000 American children. However, a South African study found the Beery VMI to be reliable in clinical practice and as a research tool for local children. Furthermore, no significant differences were found between groups defined on the basis of gender, socioeconomic status, language or the type of school programme for which they were enrolled. ${ }^{16}$ Formal IQ testing was not undertaken; however, the educational status of each child was determined by school progress, grade placement and ageappropriateness for the grade in which each child had been placed.
Statistical analysis was carried out using Statistica Release 8 (StatSoft Inc, Tulsa, OK, USA), employing descriptive statistics, means testing, simple correlations, frequency analysis and graphics subprogrammes.

\section{Results}

Forty parents and subjects agreed to participate: the final sample comprised 24 males and 16 females aged between 7 years 5 months and 19 years 6 months (mean 13 years 4 months \pm 3 years 5 months). BW ranged from $600 \mathrm{~g}$ to $3400 \mathrm{~g}$ (mean $1928 \pm 778 \mathrm{~g}$ ) and gestational age (GA) from 24 to 40 weeks (mean $32.4 \pm 6.3$ weeks). The reliability of parental recall was confirmed by the significant correlation between BW and GA $(r=0.861 ; p<0.001)$. Just over half the infants were born in public tertiary hospitals (23/40); however, 7 of the 11 children with a GA $<28$ weeks were delivered at private hospitals. When analysed for the group as GA v. hospital type, more low-gestation babies were treated in the private sector $(r=0.42 ; p<0.01)$. This is probably the result of private hospitals not applying the public sector's policy of non-ventilation of infants weighing $<1000 \mathrm{~g}$ at birth. ${ }^{13}$

Fig. 1 shows the subjects' GA in relation to the year of birth. In this group of children and adolescents with SD there is fairly consistent representation of infants across the GA range. The trend line shows a downward trend towards lower BW of SD subjects in the later years, but the correlation is not significant $(r=0.13 ; p=0.43)$.

The relationship between length of stay (LOS) and BW displayed in Fig. 2 clearly shows that the duration of hospitalisation was inversely related to GA. The short stays for the higher BW infants (typically < 1 week) suggest that they were asymptomatic at birth, did not require admission to higher levels of care, and were ready for discharge within a few days.

Table 1 explores the relationships between factors such as birth history, gender and chronological age and the measured outcome.

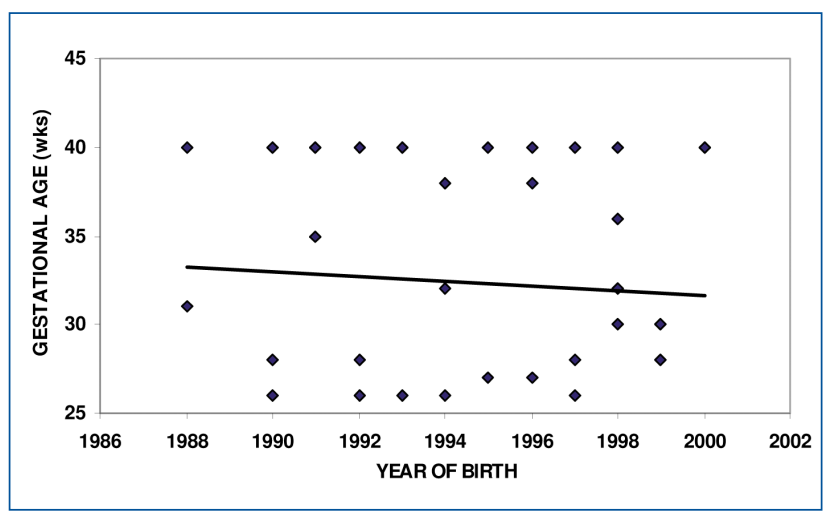

Fig. 1. Gestational age v. year of birth (40 weeks was assigned for parents to categorise infant as being 'full term').

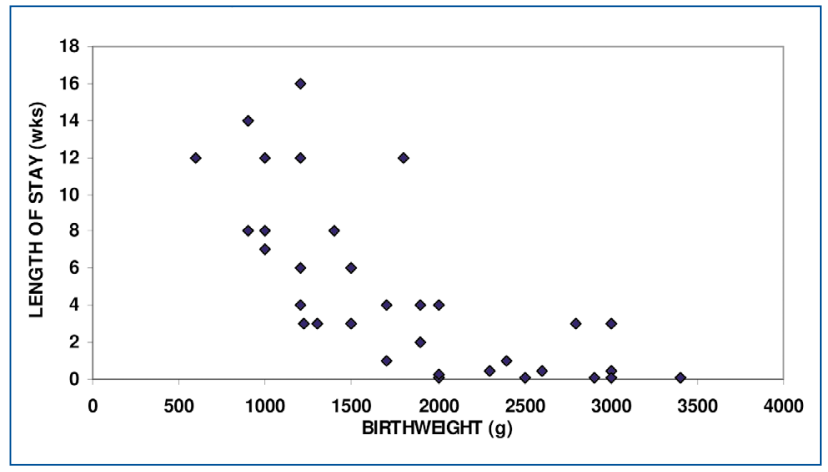

Fig. 2. Length of stay v. birth weight. 
Table 1. Relationships between participants' demographics and selected outcomes

\begin{tabular}{lcc}
\hline & Correlation coefficients $(\boldsymbol{r})$ & Significance $(\boldsymbol{p}$-value $)$ \\
\hline VIS-z and VMI-z & 0.70 & $<0.001$ \\
Gestational age and VIS-z or VMI-z & $0.14 ; 0.14$ & NS \\
Birth weight and VIS-z or VMI-z & $0.11 ; 0.16$ & NS \\
Gestational age and FMS or school grade & $0.13 ; 0.08$ & NS \\
Birth weight and FMS or school grade & $0.30 ; 0.09$ & NS \\
Male sex and FMS & 0.34 & 0.03 \\
Chronological age and VIS- $z$ & 0.36 & 0.02 \\
VIS- $z$ visual perception $z$-scores; VMI-z = visual motor impairment $z-$-scores; NS = not significant; FMS = functional mobility scale. & \\
\hline
\end{tabular}

VMI z-scores ranged from 0.2 to $<-3.7$ (mean $-2.27 \pm 1.04$ ) and VIS $\mathrm{z}$-scores ranged from -0.5 to $<-3.7$ (mean $-2.29 \pm 1.04$ ). There were no significant correlations between $\mathrm{BW}$ or GA and outcomes, i.e. irrespective of whether the study subjects were born at or before term there were no differences in FMS, VIS-Z-scores or VMI-Z-scores. However, all z-scores were low, with only one marginally above the mean for age.

There were also interesting relationships for the VIS- $\mathrm{z}$ and VMI$z$-scores. VIS scores correlated with chronological age, implying that there were improvements as the subjects grew older (Fig. 3). There were also correlations between VIS and VMI scores and school grade appropriateness, i.e. those with scores that deviated less from the norm tended to be in the appropriate class at school $(p=0.004$ for grade and VIS-z; $p=0.027$ for grade and VMI-z). Overall, 16 subjects were in age-appropriate classes, 7 were in a lower grade and 17 were in special classes with an adapted curriculum.

Functional mobility was assessed in all subjects, with 20 found to be walking independently in both school and community settings (FMS ratings 5 and 6). The remainder used walking aids or wheelchairs (FMS 1 - 4). There was a significant correlation between male sex and FMS, particularly at the level of independent walking. It is important to note that the SD and any associated abnormalities were nonprogressive, eliminating the likelihood of underlying neurological diseases or conditions.

\section{Discussion}

Contrary to expectation we found that in a select group of children and adolescents with SD: (i) both term and preterm infants were represented (almost equally); and (ii) there was not a diminishing contribution from the preterm group over time. This does not imply that term and preterm infants are at equal risk for PVL and SD; the risk is significantly higher in the preterm group (representing $<3 \%$ of births) and very much lower for term infants who represent the overwhelming majority of births. Others

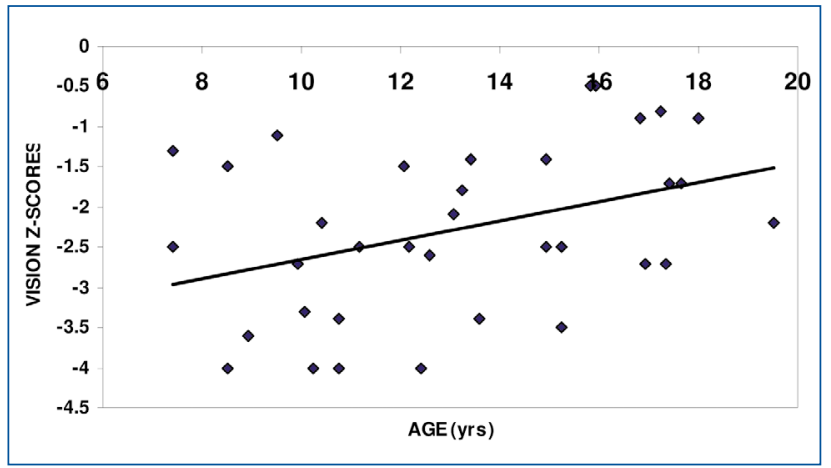

Fig. 3. Vision $z$-scores v. age. have also commented on this finding of SD in infants of both term and preterm pregnancies in follow-up and outcome studies involving SD patients. ${ }^{5,17,18}$ However, it is important to note since health practitioners and therapists treating such children commonly consider that SD is usually a consequence of prematurity.

Our results also strongly suggest that the term infants were asymptomatic at birth, for the most part being discharged from hospital within $3-4$ days. This is consistent with evidence that the neurological insult may occur prenatally, after which the fetus 'accommodates' the insult and birth subsequently proceeds normally without evidence of asphyxia or the need for resuscitation. ${ }^{7}$ On the other hand preterm infants are usually exposed in the more-immediate perinatal period and run the risk of respiratory distress, intensive care, assisted ventilation and the complication of intraventricular haemorrhage. ${ }^{7}$ While the brain insult might have occurred under different circumstances in our two subgroups, there were no correlations between functional outcomes and BW or GA of the affected children and adolescents. In other words the clinical outcomes were similar whether the infants were born at or before term, or had suffered their neurological insults earlier in utero or later at or around the time of delivery. Some of these results differ from others that have shown similarities in later cognitive functioning for term and preterm SD subjects but more visuoperceptual problems in those born preterm. ${ }^{19}$ In our study the VMI-z and VIS-z-scores were slightly lower for the preterm than the for the term group (means of $-2.35 \mathrm{v} .-2.1$ ), but this was not statistically significant. Therefore this difference from the literature might have been the result of a relatively small sample size.

All the children in the study had VMI and VIS impairments; however, cognisance must be taken of the fact that the focus was on a group of children with special physical and educational needs who had access to special schools. This almost certainly resulted in bias, and the sample probably represents a group with significant $\mathrm{SD}$ and associated developmental impairment. There are probably other SD children and adolescents within the community, most of whom probably function at normal or near-normal levels, ${ }^{20,21}$ while a minority might be too severely affected to warrant care in special education facilities.

PVL (associated with SD) is the principal cause of visual impairment in children born prematurely. ${ }^{22}$ In this study VIS (but not VMI) correlated significantly with chronological age, perhaps because VIS may improve with age as a result of habituation and experience. Cortical visual impairment has been found to improve over time. ${ }^{23}$ Visuomotor tasks are more complex and involve a motor component, an aspect that could be problematic in these children who probably also have fine-motor impairment. ${ }^{24}$ The significant correlation between visuomotor and VIS scores and grade 
appropriateness indicates that these visual impairments can adversely affect school performance, particularly in respect of reading, writing and mathematics. ${ }^{24}$ Recent studies suggest that more effort must be directed towards diagnosis and management of the visual dysfunction in survivors of PVL. ${ }^{19,25}$ These barriers to learning require specialised teaching strategies and skills. The children in this group also demonstrated considerable limitations in mobility at school and in the community. Their need for walking aids and wheelchairs highlights the importance of an accessible school environment.

Key observations of this study include: clinically significant SD is a problem found in children and adolescents irrespective of duration of pregnancy; it is likely to be the result of perinatal events in patients born prematurely, and earlier intrauterine events in those born at or near term; preterm infants continue to be at risk despite advances in neonatology over the past decades; SD is associated with significant problems in the areas of VIS and VMI.

\section{References}

1. Erken EHW, Bischof FM. Iliopsoas transfer in cerebral palsy: the long-term outcome. J Pediatr Orthop 1994;14:295-298.

2. Bischof FM, Erken EHW. Hip subluxation and dislocation in cerebral palsy: assessment and 2. Bischof FM, Erken EHW. Hip subluxation and
management. S Afr J Bone Joint Surg 1996; VI (6);6-8.

3. Bischof F, Basu D, Pettifor JM. Pathological long bone fractures in residents with cerebral palsy in a 3. Bischof F, Basu D, Pettifor JM. Pathological long bone fractures in residents with
long-term care facility in South Africa. Dev Med Child Neurol 2002;44:119-122.

4. Mutch L, Alberman E, Hagberg B, Kodama K, Velickovic Petat M. Cerebral palsy epidemiology: where are we now and where are we going? Dev Med Child Neurol 1992;43:547-555.

5. Krageloh-Mann I, Petersen D, Hagberg G, Vollmer B, Hagberg B, Michaelis R. Bilateral spastic cerebral palsy - MRI pathology and origin: analysis from a representative series of 56 cases. Dev Med Child Neurol 1995;37:379-397.

6. Goddard-Finegold J. Perinatal aspects of cerebral palsy. In: Miller G, Clark GD. The Cerebral Palsies: Causes, Consequences and Management. Boston: Butterworth Heinemann, 1998:151-171.

7. Volpe JJ. Hypoxic ischaemic encepalopathy - neuropathology and pathogenesis. In: Neurology of the Newborn. 5th ed. Philadelphia: Saunders, 2008:347-399.
8. Wu YW, Croen LA, Shah SI, Newman TB, Najiar DV. Cerebral palsy in a term population: risk factors and neuroimaging findings. Pediatrics 2006;118:690-697.

9ugumotoT, Woo M, Nishida N, et al. When do brain abnormalities in cerebral palsy occur? An MRI study. Dev Med Child Neurol 1995;37:285-292.

10. Davies VA, Rothberg AD, Ballot DE. The introduction of surfactant replacement therapy into South Africa. S Afr Med J 1995;85:637-640

11. Saigal S, Doyle LW. An overview of mortality and sequelae of preterm birth from infancy to childhood. Lancet 2008;371:261-269.

2. Wood NS, Marlow N, Costeloe K, Gibson AT, Wilkinson AR. Neurologic and developmental disability after extremely preterm birth. N Engl J Med 2000; 343:378-384.

13. Smith J, Pieper CH, Kirsten GF. Born too soon, too small to die - a plea for a fair innings. S Afr Med J 1999:89:1148-1151.

14. Graham HK, Harvey A, Rodda J, Nattrass GR, Pipiris M. The Functional Mobility Scale (FMS). J Pediatr Orthop 2004; 24: 514-520.

15. Beery KE, Buktenica NA. Developmental Tests of Visual-Motor Integration and Visual Perception. Cleveland: Modern Curriculum Press, Schuster and Schuster, 1998.

16. Dunn M, Loxton H, Naidoo A. Correlations of scores on the developmental test of visual-motor integration and copying test in a South African multi-ethnic preschool sample. Percept Mot Skills 2006;103:951-958.

17. Bax M, Tydeman C, Flodmark O. Clinical and MRI correlates of cerebral palsy: the European cerebral palsy study. JAMA 2006;296:1602-1608.

18. Volpe JJ. Hypoxic ischaemic encepalopathy - clinical aspects. In: Neurology of the Newborn. 5th ed. Philadelphia: Saunders, 2008:400-480.

19. Pagliano E, Fedrizzi E, Erbetta A, et al. Cognitive profiles and visuoperceptual abilities in preterm and term spastic diplegic children with periventricular leucomalacia. J Child Neurol 2007;22:282-288. 20. Roze E, van Braeckel KN, van der Veere CN, et al. Functional outcome at school age of preterm infants with periventricular hemorrhagic infarction. Pediatrics 2009;123:1493-1500.

21. Bassan H, Limperopoulos C, Visconti K, et al. Neurodevelopmental outcome in survivors of periventricular hemorrhagic infarction. Pediatrics 2007;120:785-792.

22. Jacobson LK, Dutton GN. Periventricular leucomalacia: an important cause of visual and ocular motility dysfunction in children. Survey Ophthalmol 2000;45:1-12.

23. Dutton GN. Cognitive vision, its disorders and differential diagnosis in adults and children: knowing where and what things are. Eye 2003;17: 289-304
whece

24. Schneck CM. Visual perception. In: Case-Smith J. Occupational Therapy for Children, 5th ed. St Louis: Elsevier Mosby, 2005:412-448.

25. Fazzi E, Bova S, Giovezana A, et al. Cognitive visual dysfunctions in preterm children with periventricular leucomalacia. Dev Med Child Neurol 2009, March 9. [Epub ahead of print] PMID: 19416337 [PubMed - as supplied by publisher]

Accepted 9 November 2011. 